\title{
Análisis de la situación del Abandono Temprano de la Educación y Formación en Castilla y León
}

\section{Analysis of the Early School Leaving situation in Castile and Leon}

\author{
María Luz Martínez Seijo \\ luz.martinez@gmail.com \\ LAURA RAYÓn Rumayor ${ }^{1}$ \\ laura.rayon@uah.es \\ Juan Carlos Torrego Seijo \\ juancarlos.torrego@uah.es \\ Universidad de Alcalá, España
}

\section{Resumen:}

El presente estudio identifica las medidas previstas por la Administración de Castilla y León entre los años 2007-2011 para la prevención y lucha contra el abandono temprano de la educación y la formación, analiza qué concepción tiene el profesorado sobre el fenómeno, qué sabe sobre dichas medidas y cómo valora su grado de implantación y efectividad. Esta comunidad destaca por sus buenos resultados en la evaluación internacional PISA, la evaluación Diagnóstico, y por ser una de las CCAA que más medidas ha arbitrado para prevenir y combatir el abandono temprano. Pero su tasa desde el 1998 roza la media nacional y la sobrepasa en el 2011. Tras un análisis documental sobre las medidas contempladas en los Convenios y documentos relacionados con el ATEF, se diseña un cuestionario de evalua-

\begin{abstract}
:
This study identifies the measures provided by the government of Castile and Leon in the years 2007-2011 to prevent and combat early school leaving. It also conceptualises teachers' views about the phenomenon, what they know about these measures and how they rate their degree of implementation and effectiveness. This community is known for its educational success considering international benchmarks such as PISA assessment or diagnosis evaluations. However, its dropout rate has touched the national average since 1998 and went over it in 2011. After a documentary analysis of the measures referred to in the Agreements with the Ministry of Education and related documents on ESL, we designed an evaluation questionnaire validated by experts in order to know the implementation of the measures
\end{abstract}

1 Dirección para correspondencia (correspondence address):

Laura Rayón Rumayor. Dpto. de Ciencias de la Educación. Facultad de Educación. Universidad de Alcalá. C/ Madrid, 1. 19001, Guadalajara (España). 
Análisis de la situación del Abandono Temprano de la Educación y Formación en Castilla y León

María luz Martínez Seijo, laura Rayón Rumayor y Juan Carlos Torrego Seijo

ción de expertos para conocer la valoración del profesorado sobre la ejecución de medidas; otro cuestionario para una muestra de 67 centros en CyL, y un grupo de discusión. Los resultados obtenidos permiten contrastar la visión institucional y la del profesorado, dimensión clave para la movilización y articulación de las medidas en la prevención y paliación del abandono temprano. Concluimos reflexionando sobre la necesidad de realizar un mayor esfuerzo en la ejecución de medidas, ampliar la coordinación con otras Consejerías y agentes externos al ámbito educativo, dar más apoyo a los centros y realizar una amplia difusión del problema del ATEF y sus consecuencias educativas, económicas y socio-laborales.

\section{Palabras clave:}

Abandono temprano de la educación; medidas, programas y convenios educativos; profesorado. to combat early school leaving; another questionnaire with a sample of $67 \mathrm{High}$ Schools schools in Castile and Leon and a group discussion were also used. Results allow us to contrast institutional and teachers' views, a key dimension for the mobilisation and coordination of measures for the prevention and mitigation of ESL. Our study concludes that further efforts are to be made in the implementation of measures, in improving coordination with other ministries and agents outside the field of education, in giving more support to schools, and in performing a wide dissemination of the problem and its educational, economic, social and labour consequences.

\section{Key words:}

Early school leaving; educational measures, programmes and agreements; faculty.

\section{Résumé:}

La présente étude cherche à identifier les mesures prévues par l'Administration de Castille-et-Léon entre 2007 et 20011 pour la prévention et la lutte contre I'abandon scolaire précoce, à analyser comment le phénomène est-il perçu par les professeurs, la connaissance qu'ils ont de ces mesures ainsi que leurs opinions sur leur mise en place et leur efficacité. Castille-et-Léon se distingue par I'obtention de bons résultats dans le cadre de l'évaluation internationale PISA, de l'évaluation diagnostique et pour être une des communautés autonomes ayant pris le plus de mesures pour prévenir et lutter contre l'abandon scolaire précoce. Cependant, son taux, depuis 1998, frôle celui de la moyenne nationale et ne le dépasse qu'en 2011. Après avoir analysé les mesures envisagées dans les Conventions et les documents relatifs à l'ASP, nous avons élaboré un questionnaire d'évaluation d'experts afin de cerner la perception de l'ensemble des professeurs sur la mise en place de ces mesures ; un autre questionnaire à l'attention de 67 établissements scolaires de C-et-L ainsi qu'un groupe de discussion. Les résultats obtenus nous permettront de contraster la vision institutionnelle avec celle de l'ensemble des professeurs, une étape clé pour une meilleure mobilisation et articulation des mesures dans le cadre de la prévention et de la diminution de l'abandon scolaire précoce. Nous conclurons par une réflexion sur les nécessités d'un plus grand effort dans la mise en place de mesures, d'une plus ample coordination entre d'autres Consejerías et des agents extérieurs au domaine éducatif, d'un soutien majeur aux établissements et d'une plus grande diffusion des problèmes de l'ASP et de ses conséquences éducatives, économiques et socio-professionnelles.

Mots clés:

Abandon scolaire précoce; mesures, programmes et conventions éducatives; professeur.

Fecha de recepción: 9-9-14

Fecha de aceptación: 26-3-2015 


\section{Introducción}

Para la Comisión Europea (2014) el abandono prematuro representa un desafío complejo a nivel individual, nacional y europeo. Para hacer frente a esta situación, es vital reconocer que, si bien el abandono prematuro es, en apariencia, un problema para los sistemas de educación y formación, sus causas subyacentes están incrustadas en mayores contextos sociales y políticos.

El problema del abandono suele confundirse con el fracaso escolar, y algunos estudios relacionan ambas problemáticas, introduciendo el fracaso escolar como factor determinante para el ATEF² (Fernández Enguita et. al., 2010; Calero et al, 2010; González et al. 2013). Las causas son variadas, de tipo personal y familiar (Roca, 2010; Espínola y Claro, 2010; Comisión Europea, 2014), referidas a la articulación entre contexto socioeconómico, laboral e instructivo (Alegre, 2010; Poy, 2010; Marina, 2011; Alemany et al. 2013), la clase social de procedencia (Bernardi y Requena, 2010), y las directamente relacionadas con el sistema escolar, destacando la variable "ayudas económicas" (Alegre, 2010), la perspectiva de la Orientación en los centros en los que prevalece la opción del Bachillerato, incluso para alumnos con dificultades en la ESO, y la poca flexibilidad del sistema en la oferta de itinerarios formativos, ampliamente analizada en el trabajo citado de Fernández Enguita et. al. (ibídem.)

En este trabajo la definición del ATEF viene dada por la Cumbre de Lisboa y se va a referir en Europa al:

"porcentaje de jóvenes entre los 18 y los 24 años que no ha alcanzado una titulación de enseñanza secundaria superior y se encuentra fuera del sistema educativo, o de los sistemas de formación, en las últimas cuatro semanas antes de ser encuestados por la EPA"3.

Nos encontramos con una definición técnica del problema, y con la reducción del ATEF como uno de los objetivos europeos definidos en 2010 y previstos para el 2020 en la agenda europea.

2 ATEF: Abandono temprano de la educación y formación.

3 EPA: Encuesta de Población Activa. 
Análisis de la situación del Abandono Temprano de la Educación y Formación en

Castilla y León

María luz Martínez Seijo, Laura Rayón Rumayor y Juan Carlos Torrego Seijo

\section{El abandono temprano de la educación y formación en España}

La situación del ATEF en Europa es dispar, hay países ${ }^{4}$ que han logrado estar por debajo del 10\%, otros como España superan el 21\% (INE, 2015). La implantación de la $\operatorname{LOGSE}^{5}$ y la obligatoriedad de la educación hasta los 16 años suponen una reducción en la tasa de abandono, bajando en 10 puntos desde la década de los 90 a la del 2000 (tabla 1). Pero, es importante advertir que en la comparativa entre el abandono y los resultados en el informe PISA en algunas $\mathrm{CCAA}^{6}$, hay una tremenda disparidad en el índice de abandono temprano, con grandes diferencias entre ellas (tabla I). Ello debería implicar más investigación, sobre todo en las CCAA más afectadas, pero no es la tendencia, dado el escaso número de investigaciones al respecto (Mora 2010; Serra y Paluadárias, 2010; Alemany et al.,2013; González et al., 2014). Ante su gravedad como problema educativo-social, el abandono escolar comienza a tener importancia y ser objeto de estudio desde el año 2003, aunque por distintas razones es un tema todavía poco investigado (Velaz de Medrano y de Paz, 2010).

Tabla 1. Abandono educativo temprano: Población de 18 a 24 años que no ha completado el nivel de E. Secundaria $2^{a}$ etapa y no sigue ningún tipo de educación-formación.

\begin{tabular}{lccccccccc}
\hline CCAA & $\mathbf{1 9 9 8}$ & $\mathbf{2 0 0 3}$ & $\mathbf{2 0 0 6}$ & $\mathbf{2 0 0 8}$ & $\mathbf{2 0 0 9}$ & $\mathbf{2 0 1 0}$ & $\mathbf{2 0 1 1}$ & $\mathbf{2 0 1 2}$ & $\mathbf{2 0 1 3}$ \\
\hline Andalucía & 38.6 & 38.2 & 36.9 & 38.5 & 37.5 & 34.7 & 32.5 & 28,8 & 28,4 \\
Aragón & 22.8 & 24.0 & 28.7 & 25.6 & 25.1 & 23.7 & 22.7 & 20,4 & 18,8 \\
Asturias & 24.5 & 28.6 & 22.3 & 19.7 & 21.1 & 22.3 & 21.9 & 19,4 & 18,7 \\
Balears (Illes) & 38.1 & 41.4 & 34.9 & 43.2 & 40.8 & 36.7 & 30.7 & 30,1 & 29,7 \\
Canarias & 34.5 & 32.7 & 34.8 & 34.1 & 31.2 & 30.4 & 31.5 & 28,4 & 28,3 \\
Cantabria & 25.8 & 29.1 & 24.0 & 22.8 & 23.9 & 23.9 & 21.2 & 14,0 & 11,8 \\
Castilla y León & 23.4 & 24.4 & 24.4 & 26.0 & 27.0 & 23.3 & 27.3 & 21,5 & 18,8 \\
Castilla-La Mancha & 39.0 & 36.2 & 38.0 & 38.1 & 34.4 & 33.2 & 31.6 & 26,9 & 26,6 \\
Cataluña & 30.1 & 33.9 & 27.0 & 33.2 & 31.9 & 29.0 & 26.0 & 24,0 & 24,4 \\
Ceuta y Melilla & 40.8 & 40.8 & 45.7 & 42.1 & 36.0 & 40.7 & 40.4 & 37,0 & 35,3 \\
C. Valenciana & 36.2 & 36.0 & 30.4 & 33.1 & 32.8 & 29.2 & 27.4 & 26,9 & 22,3
\end{tabular}

4 Países como Noruega, Polonia, República Checa, Eslovaquia, Dinamarca, Croacia, Suecia, Finlandia, Austria, o Lituania han logrado alcanzar el objetivo europeo de reducir la tasa de ATEF a menos del $10 \%$, en el extremo contrario aparecen Malta, Portugal, España, o Islandia con porcentajes superiores al 25\%.

5 LOGSE: La Ley Orgánica General del Sistema Educativo.

6 CCAA: Comunidades Autónomas. 


\begin{tabular}{llllllllll}
\hline CCAA & $\mathbf{1 9 9 8}$ & $\mathbf{2 0 0 3}$ & $\mathbf{2 0 0 6}$ & $\mathbf{2 0 0 8}$ & $\mathbf{2 0 0 9}$ & $\mathbf{2 0 1 0}$ & $\mathbf{2 0 1 1}$ & $\mathbf{2 0 1 2}$ & $\mathbf{2 0 1 3}$ \\
\hline Extremadura & 40.7 & 36.9 & 35.7 & 33.6 & 34.5 & 31.7 & 29.6 & 32,2 & 28,5 \\
Galicia & 30.5 & 25.0 & 25.8 & 24.1 & 26.0 & 23.1 & 20.8 & 23,1 & 20,4 \\
Madrid & 21.0 & 22.6 & 25.3 & 26.9 & 26.3 & 22.3 & 19.8 & 22,2 & 20,3 \\
Murcia (Región de) & 39.2 & 43.0 & 39.1 & 41.0 & 37.3 & 35.5 & 30.7 & 27,7 & 26,9 \\
Navarra & 18.5 & 20.8 & 12.6 & 19.2 & 19.8 & 16.8 & 12.0 & 13,3 & 12,8 \\
País Vasco & 18.5 & 15.8 & 14.5 & 14.7 & 16.0 & 12.6 & 13.0 & 11,5 & 8,8 \\
Rioja (La) & 30.2 & 34.7 & 26.4 & 37.2 & 32.7 & 28.1 & 30.2 & 22,8 & 21,3 \\
\hline TOTAL & $\mathbf{3 0 . 8}$ & $\mathbf{3 1 . 6}$ & $\mathbf{2 9 . 9}$ & $\mathbf{3 1 . 9}$ & $\mathbf{3 1 . 2}$ & $\mathbf{2 8 . 4}$ & $\mathbf{2 6 . 5}$ & $\mathbf{2 4 , 9}$ & $\mathbf{2 3 , 5}$ \\
\hline
\end{tabular}

Fuente: EPA. INE. Elaborado propia con metodología Eurostat e informes del Ministerio de Educación, Datos y Cifras de últimos cursos.

\section{El abandono temprano en Castilla y León}

La situación del ATEF es relativa en $\mathrm{CyL}^{7}$ según los parámetros utilizados. Puede considerarse negativa al comparar su tasa con la media nacional, 27,3 y $26,5 \%$ respectivamente en el año 2011 , y si el criterio utilizado es el objetivo europeo de reducir a un $10 \%$ la tasa de ATEF para el año 2020, de optimismo si se tiene en cuenta la previsión de medidas para combatirlo. Pero no puede considerarse positiva si nos ceñimos a su evolución en los últimos años, puesto que los únicos decrementos significativos se han producido en los años 2012 y 2013, situando a CyL con el $18,8 \%$, por debajo de la media nacional del $23,5 \%$. El decremento se produce también en otras CCAA, pudiendo a atribuirse a varias razones: alto nivel de paro juvenil que favorece el retorno a los estudios de un sector que abandonó ante las buenas perspectivas laborales, o a las medidas acordadas con el MEC que se están llevando a cabo en toda España, aunque de manera desigual en planificación y amplitud de áreas abordadas.

Es preocupante dados los picos experimentados en la tasa de abandono en contraste con otras CCAA, en donde la tendencia es de reducción. Las CCAA que lo han incrementado o reducido mínimamente son pocas: la Comunidad de Madrid aumenta de 21 a 22,2\%, Aragón reduce de 22,8 al 20,4\%, Ceuta y Melilla dos décimas, o CyL con una reducción de 23,4 a $21,5 \%$. Por el contrario, las CCAA que consiguen reducir este porcentaje de una manera significativa en estos últimos doce años, son Castilla la Mancha en 13 puntos, Región de Murcia en 12, Cantabria

7 CyL: Castilla y León. 
Análisis de la situación del Abandono Temprano de la Educación y Formación en

Castilla y León

María luz Martínez Seijo, Laura Rayón Rumayor y Juan Carlos Torrego Seijo

en 11, o Andalucía y Comunidad Valenciana con una reducción de 10 puntos (Tabla 1).

\section{Objetivos del estudio}

Estos datos justificarían estudios orientados a identificar el estado del ATEF en CyL como una aproximación imprescindible con vocación descriptiva y global para mejorar las acciones preventivas y de reducción del ATEF basadas en evidencias científicas. Este estudio parte de un interrogante clave: por qué una de las CCAA que mayor número de medidas y de áreas ha incluido en los convenios firmados con el Ministerio de Educación entre 2007-2011, no tienen el impacto deseado. Abordamos el problema del ATEF atendiendo a: 1) identificar las medidas previstas por la Administración autonómica para prevenirlo y combatirlo en los años 2007-2011 en CyL, como paso imprescindible para 2) analizar qué conocimiento tiene el profesorado sobre el ATEF, las medidas arbitradas y la efectividad que las otorgan, y posteriormente, con los datos obtenidos, 3) identificar y contrastar la visión institucional y la del profesorado, para por último, 4) encontrar respuestas que expliquen por qué en esta Comunidad, siendo una comunidad con numerosas medidas, la evolución del ATEF no es halagüeña.

\section{Método y muestra}

La investigación se articula en tres momentos, en primer lugar, se analiza la ejecución de las medidas previstas por la Consejería de Educación recogidas en los convenios con el MEC entre el 2007-2011, agrupadas posteriormente en 4 bloques según su temática para su valoración por 4 grupos de distintos profesionales de la educación según campos de profesionalidad: Directores de IES, Directores de Centros de Adultos (CEPA), Orientadores y Profesorado de Formación Profesional. Este procedimiento se orienta a comprobar qué sentido tenían dichas medidas para cada uno de los colectivos profesionales, y si se ajusta a su marco valorativo consciente. Los datos se analizan en orden a identificar unidades que mejor definan las distintas medidas, construir un cuestionario que se ajuste lo más posible a unos significados compartidos, y que en 
consecuencia tuviera una estructura en los protocolos e ítems con sentido para los respondientes (Cohen et. al. 2011:378-379). En segundo lugar, con los datos obtenidos, elaboramos un cuestionario para conocer cómo se percibe el ATEF, enviados a 392 centros: 229 públicos (IES, IESO y CEO) y 163 concertados de las 9 provincias de CyL, con una muestra total de 25 centros rurales, 22 urbanos y 20 concertados (18 de ámbito urbano), con una participación del $17,1 \%$ y con un mínimo de 5 centros por provincia que garantiza la heterogeneidad territorial y diversidad de contextos educativos, cumplimentado en zonas urbanas por 15 directores, 6 Jefes de Estudio, y 2 Orientadores, y en zonas rurales por 11 directores y 12 Jefes de Estudios. El análisis de los datos del cuestionario es descriptivo, no se trata de una escala, sino de indagar de forma extensa en las creencias y valoraciones sobre la concepción del ATEF y la valoración de las medidas llevadas a cabo, tal y como lo proponen los autores más arriba citados. En un tercer momento, para garantizar la reconstrucción discursiva de los datos obtenidos anteriormente y proporcionar un control y comprobación de dichos resultados, se utiliza un

grupo de discusión. Éste queda conformado por un Director de CEPA, un Director de IES urbano en zona social de clase media, un profesor de FP de un centro situado en zona desfavorecida y con amplia experiencia en programas educativos, un Jefe de Estudios de un centro en zona rural desfavorecida y con alto índice de fracaso y abandono escolar, y un Inspector de Educación. Para garantizar también la triangulación de perspectivas y redundancia discursiva se realiza también una entrevista en profundidad al máximo representante de la Administración educativa.

\section{Resultados y discusión}

Teniendo en cuenta los objetivos del estudio, los datos obtenidos se han agrupado en tres categorías definitorias de los resultados más relevantes sobre la concepción del ATEF, las medidas aplicadas, y la tasa de ATEF y su gravedad. Se utilizan también datos estadísticos socio-demográficos y educativos en la discusión de los datos con el objetivo de poner en contexto determinadas explicaciones y reflexiones a la luz de los datos recogidos. Comprender el estado del ATEF desde esta perspectiva es coherente con la naturaleza polifacética del problema explicada más arriba, y proporciona además evidencias a partir de las cuales identificar 
Análisis de la situación del Abandono Temprano de la Educación y Formación en

Castilla y León

María luz Martínez Seijo, Laura Rayón Rumayor y Juan Carlos Torrego Seijo

futuras líneas de trabajo en los centros educativos y cuestiones sobre las que seguir indagando desde una perspectiva multidimensional del problema ${ }^{8}$.

\section{Concepción del ATEF}

El profesorado lo considera un problema grave que atribuyen al sistema educativo. No existe acuerdo respecto a qué sexo afecta más, un 64\% de los centros lo consideran un problema de ambos sexos, aunque las tasas de la EPA y estudios de referencia demuestran que afecta más a los hombres que a las mujeres, al igual que el fracaso escolar. Respecto al conocimiento sobre las medidas para abordar el ATEF se constata confusión, y en general un desconocimiento sobre las mismas. El ATEF es un problema educativo considerado relativamente nuevo por el profesorado. El Colectivo Lorenzo Luzuriaga (2012) considera que para los docentes aparece como un elemento perturbador en un contexto de clasificaciones y evaluaciones internacionales tipo PISA (2012:31). Aunque el primer Convenio de CyL firmado con el MEC data del 2007, punto de inflexión para empezar a trabajar en el problema, se señalan épocas anteriores, como el trabajo en Ios CEPA que consideran una política constante en la historia educativa de CyL para combatir el ATEF.

Datos que indicarían la necesidad de: a) caracterizar la distribución desigual por sexo, y sus consecuencias obvias, un riesgo mayor de abandono y probabilidad de que los hombres pasen a formar parte de las bolsas de exclusión social al encontrarse con serias dificultades en el acceso al mercado de trabajo; b) lo que permitiría reforzar los IES con actuaciones de prevención coherentes con dicha distribución que eviten una desvinculación progresiva; c) y en consecuencia, hacer hincapié en que Ios CEPA conforman una oferta formativa de segunda oportunidad como respuesta a los retornados a la educación y la formación.

Sobre las razones que explicarían la existencia del ATEF hay elevado consenso en destacar el fracaso escolar e historial de repetición como el más determinante $(78 \%)$, seguido por la falta de apoyo familiar al estu-

8 A partir de los resultados y la prospectiva consecuente de este estudio, se está llevando a cabo una investigación en curso "Estudio del Abandono temprano de la educación y la formación: la perspectiva de los jóvenes, familias y profesorado" bajo convenio de colaboración entre la Consejería de Educación de Castilla y León, la UAH y la UVA, bajo la dirección de Juan Carlos Torrego Seijo (UAH). 
dio $(56,2 \%)$, los problemas familiares $(45,3 \%)$, y el contexto socioeconómico $(40,6 \%)$. En el grupo de discusión, ante el reconocimiento de la naturaleza compleja y multifactorial del problema, hay acuerdo unánime sobre la necesidad de actuaciones específicas en la educación primaria, en consonancia con el Colectivo Lorenzo Luzuriaga (ibídem). Estos datos evidencian el reconocimiento del profesorado de dos antecedentes importantes para el abandono, una cuestión básica para concretar prácticas de prevención en los centros con mínimas garantías de éxito, clave también para promocionar una política educativa proactiva del "éxito para todos" desde una perspectiva inclusiva en la etapa obligatoria. Todo lo cual ayudaría a combatir la desafección y abandono de los jóvenes. Como veremos en el siguiente apartado, una dimensión imprescindible para que el Plan "Éxito Educativo" se convierta en una medida de prevención eficaz.

Otros resultados significativos son el reconocimiento de la flexibilidad escasa de nuestro sistema educativo como determinante del ATEF: sin título de la ESO no se puede continuar estudiando. Ello justificaría un debate extenso e importante sobre la conveniencia de la expedición de un título al finalizar la etapa obligatoria o la emisión de certificados de competencias para la continuidad de los estudios porque, como aspecto clave en el fracaso escolar, afecta al ATEF directamente.

\section{Las medidas de los Convenios entre la Consejería de Educación y el MEC}

No todas las medidas se han llevado a cabo, de las 43 expresadas en los Convenios, 23 no han sido ejecutadas frente a 20 ejecutadas en los 5 años analizados, con un porcentaje del $46,5 \%$ de ejecución. Las realizadas lo son por iniciativa de los centros educativos en el marco de cierto "voluntarismo". Se delega excesivamente en los centros, aunque es un problema polifacético que los centros por si solos no pueden resolver.

El Plan Regional de Prevención del Absentismo y del Abandono Escolar previsto en Convenio con el MEC en el año 2009 no se ha llevado a cabo, siendo una medida sobre la cual giran las políticas fundamentales para la reducción de la tasa de ATEF. Sus objetivos son la identificación de las bolsas de abandono y perfiles de población, definición de actuaciones concretas, y de los agentes que debieran de intervenir. Ello 
permitiría no sólo la caracterización numérica de los jóvenes que han abandonado, sino la detección sistemática de información sobre características e intereses de los posibles destinatarios de la formación, facilitando además contar con una información imprescindible para poner en común con las necesidades profesionales de la región, y generar una oferta formativa de segunda oportunidad integrada y coherente con el tejido productivo de la Región. Profesorado y administración asumen su prioridad, destacan el valor de esta actuación coordinada con otras entidades para la prevención, la identificación por diversos colectivos bien diferenciados, y una intervención sistemática cuando el abandono ha tenido lugar.

Se conocen programas (Éxito Educativo, PROA, PCPI, etc.), pero no se identifican con la lucha contra el ATEF desde un plan coordinado que abarque la prevención, la identificación de los colectivos, y acciones coordinadas con otros agentes y servicios que permitan reforzar y apoyar lo que se realiza en los centros en la línea señalada.

Aunque la Consejería reconoce la importancia de llevar a cabo un estudio sobre los casos de ATEF para identificar a los colectivos más vulnerables, intervenir específicamente en centros con mayores tasas de abandono, conocer qué variables influyen, y analizar qué tipo de incidencia tienen en el contexto geográfico, no existe un plan concreto zonal y multidisciplinar para trabajar en consonancia.

Una medida significativamente valorada por el profesorado es la FP, modalidad formativa contemplada en los Convenios para reinsertar en el sistema a los jóvenes que han abandonado. Pero hay discrepancias entre la Consejería y el profesorado, la primera destaca el papel que desempeña como formación inequívocamente orientada al mercado de trabajo, con un impulso de la oferta parcial y modular, y una oferta a distancia, aunque el profesorado sólo reconoce como ejecutadas las pruebas de acceso y cursos preparatorios para ciclos de GM y GS, destaca la ausencia de oferta modular y la escasa orientación del alumnado hacia la FP. Hay voluntad política, un discurso promocional de la FP, pero no acciones llevadas a cabo, aunque el incremento de matrícula en los últimos cursos sea evidente.

La medida más conocida, considerada específica en la atención ordinaria a la diversidad, son los PCPI. Para el Consejo Económico y Social de CyL es la medida más eficaz para luchar contra el ATEF, reconocida como tal por el profesorado, quien considera debiera ampliarse su nivel 
2 -ámbito urbano-, y extender el nivel 1 a más Centros y Ayuntamientos. Esta valoración común permitiría reforzar en los centros y profesionales una concepción del ATEF coherente con unas prácticas formativas que destierren una posible visión deficitaria del alumnado y sus familias, y coadyuve a revalorizar los IES como centros para la reincorporación de los jóvenes que han abandonado.

El Plan de Absentismo Escolar es una medida claramente relevante que implica a las Direcciones Provinciales de Educación, a los equipos directivos de centros de Primaria y Secundaria, a los CEAS, y a los agentes de servicios sociales de Ayuntamientos y Diputaciones. En el seno de la comisión del Plan de Absentismo Escolar se analizan los casos de absentismo de menores de 16 años, pero su procedimiento de actuación es considerado por el profesorado demasiado burocrático y muy lento, siendo la segunda medida considerada más ineficaz. Al respecto Marina (2011:4) expresa:

"Los planes contra el abandono escolar, para ser eficaces, deben de ser organizados por los Municipios, porque son ellos los que pueden movilizar a la ciudadanía hacia un objetivo que es conveniente para cada ciudadano y no sólo para los que tienen hijos en edad escolar".

Marina muestra proyectos en los que las administraciones locales han reducido drásticamente la tasa de fracaso y abandono escolar, como en Nueva York en la pasada década, que contaba con un $70-80 \%$ de abandono que se redujo a 20-30\%, bajo el modelo de ciudades educadoras, reuniendo a expertos de todos los campos para crear nuevas escuelas y currículum educativos.

El programa del Éxito Educativo (refuerzo extraescolar para Lengua y Matemáticas) es considerada por el Consejo Escolar y por la Consejería como el programa que ha contribuido especialmente a mejorar el éxito educativo en $\mathrm{CyL}$, factor determinante a medio plazo para reducir la tasa de ATEF. Pero el profesorado -que conoce bien el funcionamiento del programa- discrepa de su efectividad, solo un $4,3 \%$ lo consideran eficaz, mientras que es considerado como el programa más ineficaz por un $32,3 \%$. El profesorado coincide en señalar que los recursos humanos ordinarios en los centros son más importantes para poder realizar apoyos o desdobles, y cuestionan que la mejora de resultados escolares en $\mathrm{CyL}$ se deba al Programa. El éxito o fracaso escolar de los alumnos se debe a 
una diversidad de factores, atribuir la mejora exclusivamente a un programa parecería arriesgado, más aun cuando sólo se refuerzan dos asignaturas y no en todos los cursos de la ESO, ni en todos los centros.

El $\mathrm{PROA}^{9}$ es reconocido por la Consejería como específico para la lucha contra el ATEF pero no como prioritario por el profesorado que en su mayoría lo considera poco eficaz, se conoce poco porque no se aplica en todos los centros.

La formación dirigida a familias y población escolar en riesgo de abandono es otra línea de discrepancia constatada. Según la Consejería se ha realizado una formación importante para las familias ${ }^{10}$, pero el profesorado asume que hay que mejorarla. Es relevante señalar que la mayor dificultad que reconocen para tratar el problema del ATEF es la participación de las familias. El profesorado valora el entorno familiar como una de las causas más importantes del ATEF, y a la familia como la mayor dificultad para abordar el problema. Este colectivo valora unánimemente la no ejecución de medidas al respecto.

Los Planes de Mejora y Proyectos de Innovación, es otra línea discrepante. Dentro de las medidas que se contemplan en los Convenios con el MEC, figura el fomentar el intercambio de experiencias de calidad (años 2007 y 2008). Pero sólo ha habido dos Planes de Mejora en toda CyL en 5 años y dos Proyectos de Innovación para una Comunidad que consta de 9 provincias. La dinamización de espacios para que el profesorado intercambie ideas y acciones concretas sobre un tema tan complejo como es la reducción del ATEF, parece razonable.

La formación del profesorado es una medida planteada en los Convenios con el MEC que se ha incrementado paulatinamente según vemos en la tabla 2, pasando de 15 actividades en el curso 2007/08 a 50 en el curso 2011/2012 (BOCCYL de 7 de junio, 2012), aunque es significativo que sea percibida por dos centros como eficaz.

9 Programa de Refuerzo, Orientación y Apoyo.

10 En el 2009 se realizaron 10 Conferencias participando 307 madres/padres, 6 Escuelas de madres/padres con 103 participantes. En el 2010, 4 Conferencias con 65 personas, 7 Escuelas con 115 participantes, y 13 Escuelas de éxito educativo con 195 participantes. En el 2011, 5 Conferencias con la participación de 93 madres/padres, 9 Escuelas con 173 y 15 Escuelas de éxito educativo con 173 participantes. En el 2012 se ha realizado una Jornada de ATEF con 20 participantes. 
Tabla 2. Número de actividades y participantes en formación del profesorado sobre ATEF en los cursos 2007/08, 2008/09, 2009/2010, 2010/2011 y 2011/2012.

\begin{tabular}{lccccc}
\hline & $\mathbf{2 0 0 7 / 0 8}$ & $\mathbf{2 0 0 8 / 0 9}$ & $\mathbf{2 0 0 9 / 1 0}$ & $\mathbf{2 0 1 0 / 1 1}$ & $\mathbf{2 0 1 1 / 1 2}$ \\
\hline Actividades & 15 & 22 & 30 & 47 & 50 \\
\hline Participantes & 198 & 291 & 408 & 603 & 655 \\
\hline
\end{tabular}

Fuente: Respuesta Parlamentaria: BOCCYL, nº113, 7 de junio de 2012.

La Consejería de Educación destaca otras 11 medidas reconocidas por el profesorado para la prevención y lucha contra el ATEF como objetivo propio de los centros través de los recursos ordinarios del sistema: 1) Planes de acogida e integración, de apoyo y de refuerzo. 2) Identificación del alumnado en riesgo en $2^{\circ}$ y $3^{\circ}$ de la ESO. 3) Programa de convivencia escolar. 4) Plan de Orientación Académica y Profesional. 5) Atención individualizada y personalizada de asesoramiento académico y profesional. 6) Elaboración de Programas específicos de prevención del abandono escolar y de integración educativa para el alumnado de $3^{\circ}$ y $4^{\circ}$ de ESO. 7) Orientación Profesional al alumnado. 8) Coordinación con familias. 9) Planes de Acción Tutorial a través de "Escuelas de padres y madres". 10) Difusión y puesta en práctica de experiencias exitosas en relación al abandono escolar temprano.11) Colaboración con las Comisiones Provinciales de Absentismo. La prevención y lucha contra el ATEF se incorpora como objetivo propio a través de los recursos ordinarios del sistema, siendo los centros los responsables de incorporar a su proyecto y prácticas educativas la prevención; uno de los niveles de actuación contemplados por la política europea que requeriría afrontar explícitamente un modelo educativo y de orientación desde una perspectiva inclusiva y de cohesión social (CE, 2011). Se facilitaría de este modo una consideración ampliamente compartida: la flexibilización de las medidas teniendo en cuenta la heterogeneidad del territorio y la diversidad de centros, pero sin perder de vista un modelo marco de actuación que permitiera a un mismo tiempo priorizar planes y recursos en aquellas "zonas de abandono" afectadas especialmente por el problema. 
Análisis de la situación del Abandono Temprano de la Educación y Formación en

Castilla y León

María luz Martínez Seijo, Laura Rayón Rumayor y Juan Carlos Torrego Seijo

\section{Sobre la tasa de ATEF y su gravedad}

Hay coincidencias entre las distintas fuentes en restar importancia a la tasa de ATEF mostrada en la EPA a nivel nacional y autonómico. La tasa actual es percibida por factores que influyen en el problema antes de producirse, por lo que la franja de edad que se tiene como indicador, desestima que el abandono actual es consecuencia de lo realizado en periodos previos. Algunos estudios (Roca, 2010; Faci, 2011) restan importancia a la tasa de la EPA, pero hay que tener en cuenta que ésta no ha mejorado de manera significativa en los últimos años en $C y L$, para lo que podría haber una interpretación razonable: no se han tomado suficientes medidas y las que se han tomado no son suficientemente efectivas, que las medidas no hayan dado su fruto puede ser por varias razones y no estrictamente educativas. Una hipótesis que planteamos a la luz de los datos obtenidos, es que el problema, que es plural, se trata de manera singular. No sólo es un problema educativo, sino económico y social, con una relación estrecha con las políticas de empleo y sociales.

La crisis en nuestro país puede implicar el cuestionamiento del funcionamiento positivo de algunas medidas por la imprevisible magnitud de la crisis y la desafección actual a lo educativo. Pese a que el enfoque educativo puede dar resultados, el ATEF es un problema complejo y polifacético, con tantas caras como implicaciones tiene, señaladas al inicio del trabajo, que demandan la implicación del entorno. Las medidas analizadas son educativas, pero su efecto será menor si no se ponen en marcha otras en un escenario que impliquen e involucren a otras estructuras, agentes y ámbitos de trabajo (educación, formación, empleo y servicios sociales).

Los resultados obtenidos evidencian que algunas de las medidas previstas por la Consejería de educación implican a otras Consejerías y los Ayuntamientos, pero la crisis económica ha complicado la situación de las tradicionales ayudas económicas. Pese a que las respuestas parlamentarias indican que se han convocado para entidades privadas sin ánimo de lucro y entidades locales, bajo tres líneas principales de acción: educación de adultos, PCPI y programas para atender a jóvenes en situaciones desfavorecidas, éstas se ven reducidas en la realización de talleres de empleo, aulas taller, y en los programas de formación para jóvenes, como se deduce del incremento de la tasa de ATEF en CyL en el año 2011. La hipótesis plausible para el ámbito nacional: la crisis ha 
fomentado la vuelta a las aulas, no es válida en CyL, hay un descenso de la tasa de ATEF hasta el 2010 que remonta en el 2011.

La explicación para el incremento de la tasa de ATEF en el 2011 es que CyL antes "exportaba" jóvenes trabajadores a otras CCAA, pero ahora éstos han retornado -ante la falta de oportunidades laborales en otros lugares-, con menos posibilidades formativas (reducidas por los recortes) y laborales (reducidas por la crisis), supone un incremento en el padrón. Pero, los datos no muestran el incremento de población en los últimos años, sino que confirman la tendencia de despoblación juvenil que sufre $C y L$, reflejada en la tabla 3, problema también del 2011 en que la tasa de ATEF se dispara. El padrón sí explicaría la nueva reducción experimentada en el año 2012, al confirmar los datos que la población extranjera, que alcanza su tope en el año 2009, va perdiendo población anualmente, descendiendo la curva poblacional. Por ello, la población extranjera no explica el incremento de ATEF, ésta se reduce como lo hace el resto de la población. Estos datos indicarían nuevamente que las medidas previstas no dan los resultados esperados, y que el incremento de ATEF se podría deber a los recortes educativos, al incremento del paro juvenil, y a la escasa eficacia de las medidas.

Tabla 3. Población edad 18-24 años de Castilla y León: Años 2007-2012 y población extranjera por grupo de edad 15-24 años.

\begin{tabular}{lcccccc}
\hline & $\mathbf{2 0 0 7}$ & $\mathbf{2 0 0 8}$ & $\mathbf{2 0 0 9}$ & $\mathbf{2 0 1 0}$ & $\mathbf{2 0 1 1}$ & $\mathbf{2 0 1 2}$ \\
\hline Población 18-24 años & 197.050 & 195.611 & 190.087 & 182.824 & 176.927 & 150.736 \\
\hline Extranjeros 15-24 años & 21.301 & 28.093 & 29.234 & 27.942 & 27.122 & 25.828
\end{tabular}

Fuente: INE. Explotación Estadística del Padrón. Cifras de Población. Elaboración propia.

El paro juvenil aumenta en los últimos años, y lo hace por debajo de la media nacional, lo que no explicaría el incremento de ATEF en $C y L$, según vemos en la tabla 4 . Pero este factor podría desmotivar a los jóvenes en su continuidad en el estudio, es decir, la perspectiva de no encontrar trabajo ni con estudios, no estimula a los jóvenes a continuar su formación, interpretación compartida con Marina (2011). 
Análisis de la situación del Abandono Temprano de la Educación y Formación en

Castilla y León

María luz Martínez Seijo, Laura Rayón Rumayor y Juan Carlos Torrego Seijo

Tabla 4. Tasas de paro por distintos grupos de edad y sexo, Castilla y León y media nacional.

De 16 a 19 años

De 20 a 24 años

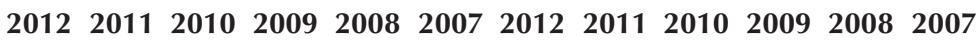

Castilla y León

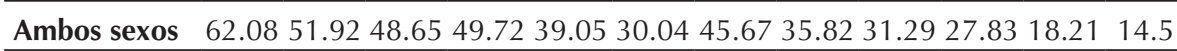

Hombres $\quad \begin{array}{llllllll}61.15 & 51.9944 .0945 .6329 .22 & 26.81 & 44.7636 .54 & 31.39 & 24.12 & 14.38 & 11.43\end{array}$

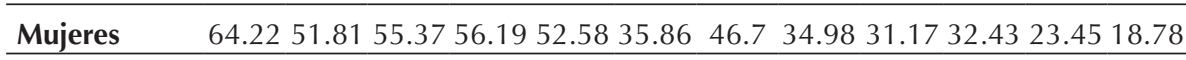

Nacional

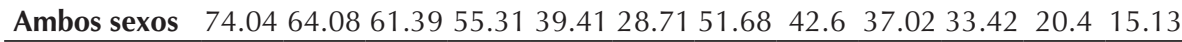

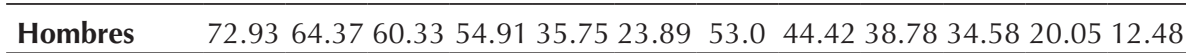

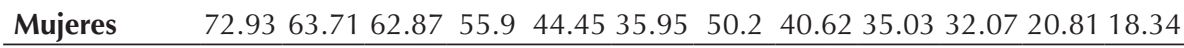

Fuente: INE. Datos de la EPA. INE. Elaboración propia.

Otra explicación que justificaría la reducción escasa de la tasa de ATEF en CyL se basa en el recorte de ayudas y subvenciones que la Consejería de Educación ha realizado desde el año 2009 a entidades privadas sin ánimo de lucro para la realización de actuaciones dirigidas a la prevención y reducción del ATEF. Según se desprende de la resolución de las convocatorias anuales, se ha pasado de 73.788 en el año 2008 a 66.410 en los siguientes tres años para no ser convocadas en el año 2012. Estas ayudas también se ven reducidas desde el año 2011 un $72,59 \%$ en el Proyecto de Ley de Presupuestos generales para la Comunidad de CyL (2012, tomo 11:94).

La reducción en los PCPI ha sido de prácticamente un millón de euros en dos años, 3.200.000 en el 2009 y 2.396.924 en el 2010 según se observa en ambas convocatorias, comunes con la de las entidades privadas sin ánimo de lucro. La situación se agrava en el 2012, con una reducción 35,6\% para las entidades sin ánimo de lucro respecto a la convocatoria 2011, a pesar de haber un incremento previsto del $4,62 \%$ (PGCCyL, 2012, tomo 11:104). Debemos recordar que un PCPI no es educación secundaria post-obligatoria, pero implica una formación/cualificación que abre la posibilidad a continuar estudios.

La reducción de posibilidades formativas tiene una repercusión clara en la no continuidad de estudios y como consecuencia en el ATEF. Es evidente que en CyL el factor crisis no ha ayudado a la vuelta a los estudios, puesto que la tasa de abandono ha aumentado considerablemente, situándola en los niveles del año 1998. La situación social no ha variado 
en los últimos años significativamente, y el porcentaje de jóvenes que abandonaba sus estudios se mantiene, creando una diferencia social estable e inamovible. La población que hay que captar está fuera de la escuela, "perdida" en la sociedad.

\section{Conclusiones}

La coordinación de diversas estructuras, agentes y ámbitos de trabajo (educación, formación, empleo y servicios sociales), junto con el compromiso de todos: familias, profesorado, sociedad y administraciones es una perspectiva compartida, clave para combatir el problema. Hay una idea clara sobre lo que se debe hacer, pero no de lo que se hace realmente. Si hay un enfoque coincidente, debe haber también una actuación determinante encaminada a ello que debe ser impulsada por la Junta de CyL, administración que asume el área social, de economía y empleo y educación. Los profesores no perciben la importancia de los agentes externos, como el Consejo Escolar, el Procurador Común, Sindicatos, AMPAS y Comunidad Escolar en la política educativa, sólo perciben como influyente al Parlamento Autonómico. Por ello, las medidas educativas son vistas como unilaterales, no compartidas o participadas con el resto de agentes. Esto implicaría una escasa rentabilidad al Ilevarlas a cabo y un menor porcentaje de probabilidad de éxito. Si una medida se asume como compartida, tiene más validez que aquella que no lo es. Sería interesante analizar hasta qué punto y en qué aspectos un problema educativo como el ATEF influyen los agentes educativos externos. Si es un problema polifacético y multidisciplinar, para abordarlo se requiere la colaboración del mayor número posible de agentes, tanto para el análisis del marco del ATEF como para tratar las medidas abordadas bajo un modelo compartido.

Un pacto educativo a nivel nacional permitiría devolver a la política educativa una proyección de consenso y una toma de decisiones participadas por los distintos representantes implicados en el ATEF. La consideración de gravedad que tiene el ATEF a nivel nacional y autonómico, legitimaría la necesidad de un pacto para abordar éste y otros problemas que afectan de una manera negativa al sistema educativo de nuestro país, y por ende al sistema socio-económico y la ciudadanía.

Todas las fuentes consultadas asumen que lo que determina el fracaso 
escolar y posteriormente el ATEF es el impacto del entorno social y económico, ratificado por los estudios revisados, e incluso el informe PISA. Todos abordan el ATEF desde una perspectiva que debe poner en relación lo educativo, con otras estructuras socio-laborales y económicas. La relación entre una baja renta per cápita, el porcentaje de promoción en la ESO y el número de alumnos que registran incidencias en el aula por provincia es inversamente proporcional. Un bajo PIB en una provincia, coincide con alto fracaso escolar y mayor porcentaje de incidencias en el aula. Esta relación deja claro que en la tasa de ATEF influyen no sólo las circunstancias de una CCAA, sino las características de una unidad inferior, la provincial. Con una política educativa común en una CCAA pluri-provincial, las diferencias educativas son claramente influenciadas en gran medida por las circunstancias socio-económicas de la zona. Obviamente, es necesario abordar una implantación de medidas diferenciadas en función de indicadores educativos y socio-económicos.

Evaluar el éxito de las medidas para combatir el ATEF y analizar los resultados no puede basarse sólo en la tasa de incremento de alumnos en la FP, las tasas brutas de graduación en enseñanzas pos obligatorias y la tasa anual de ATEF, porque estas dos fuentes son aproximativas, pero no definitivas para evaluar el éxito de unas medidas educativas. El fracaso escolar es indicador del ATEF, pero ambas tasas en CyL son muy dispares $y$, por tanto, es inexacto indicar este factor como una herramienta de evaluación, aunque la obtención del título de la ESO determine qué alumnos pueden seguir estudiando. El incremento del alumnado cursando estudios de FP tampoco se puede considerar un indicativo determinante, porque la matrícula de FP ha ido incrementándose ligera y progresivamente en los últimos años con la crisis económica. Este incremento como indicador hay que utilizarlo con cautela, puesto que los datos se mezclarían con los efectos de la crisis -un factor comentado por todas las fuentes- y ésta, sin duda, no es indicador educativo, por lo que no debiera correlacionarse el éxito de las medidas educativas con el indicador de incremento de la matrícula de FP. Ejecutar el Plan de Abandono parece prioritario porque permitiría tratar con suficiente seriedad y hacer un diagnóstico real del problema del ATEF en CyL. Finalmente, parece necesario seguir investigando desde dentro del sistema en colaboración con la comunidad educativa para comprender la amplitud, profundidad y complejidad de los procesos que afectan al ATEF, y desde la perspectiva del alumnado y familias constatar el efecto de las medidas ejecutadas. 
Análisis de la situación del Abandono Temprano de la Educación y Formación en Castilla y León

María luz Martínez Seijo, laura Rayón Rumayor y Juan Carlos Torrego Seijo

\section{Referencias bibliográficas}

Alegre, M.A. y Benito, R. (2010). Los factores del abandono educativo temprano. España en el marco europeo. Revista de Educación, número extraordinario, 65-92.

Alemany Arrebola, I., Rojas Ruiz, R., Gallardo Vigil, M.I., Sánchez Fernández, S. (2013). El abandono escolar temprano en un contexto multicultural. Análisis de sus causas por los agentes profesionales y sociales implicados, Journal for Educators, Teachers and Trainers JETT, Vol. 4 (2); ISSN: 1989-9572, pp. 191-203.

Bernardi, F. y Requena, M. (2010). Inequality in Educational Transitions: the case of postcompulsory education in Spain, Revista de Educación, número extraordinario, 93-118.

BOCCYL, VIII Legislatura, Contestación de la Junta de Castilla y León a la Preguntas Escrita: 0802324, formulada por el Procurador Don Francisco Ramos Antón relativa al abandono escolar temprano y formación del profesorado, $n^{\circ}$ 113/8, 07 de junio de 2012, 17039.

Calero, J, Choi, A, y Waisgrais, S. (2010). Determinantes del riesgo de fracaso escolar en España: una aproximación a través de un análisis logístico multinivel aplicado a PISA-2006. Revista de Educación, número extraordinario, 225-256.

Cohen, L.; Manion, L. y Morrison, K. (2011). Research Methods in Education. London: Taylor \& Francis. ( $7^{\text {a }}$ edición)

Colectivo Lorenzo Luzuriaga, (2012). Documento sobre el fracaso escolar en el estado de las Autonomías, Madrid, 51.

Comisión Europea/EACEA/Eurydice/ Cedefop. (2014). Tackling Early Leaving from Education and Training in Europe: Strategies, Policies and Measures. Eurydice and Cedefop Report. Luxembourg: Publications Office of the European Union.

Espínola Hoffman,V. y Claro Stuardo, J.P. (2010). Estrategias de prevención de la deserción en la Educación Secundaria: perspectiva latinoamericana, Revista de Educación, número extraordinario, 257-280.

Faci Lucía, F., (2011). El Abandono Escolar prematuro en España, Revista de la asociación de Inspectores de Educación de España, no 14. Disponible en: http://www. adide.org/revista/index.php?option=com_content\&task=view\&id=282 \&ltemid=70 [consulta 2015, 27 de febrero]

Fernández Enguita, M; Mena Martínez, L; y Riviere Gómez, J. (2010). Desenganchados de la educación: procesos, experiencias, motivaciones y estrategias del abandono y del fracaso escolar, Revista de Educación, número extraordinario, 119-145.

Fernández Macías, E., Muñoz de Bustillo Llorente, R., Braña Pino, F.J. y Antón Pérez, J.I. (2010). Algunas apreciaciones aritméticas sobre el fracaso y el abandono escolar en España, Revista de Educación, número extraordinario, 307-324.

González, S., Cabrera, M.C, García, M.P., Muñoz, J.M, Pozo, C., Ruiz ,F., Triviño M.A. (2013).Alumnado en riesgo de abandono. Análisis de los factores contextuales, Fundación Centro de Estudios Andaluces, Consejería de la Presidencia, Junta de Andalucía, Sevilla.

INE, Explotación Estadística del Padrón. Cifras de Población. Disponible en http://www. ine.es/jaxi/menu.do?type=pcaxis\&path=/t20/e245/\&file=inebase [consulta 2012, 1 de junio] 
Análisis de la situación del Abandono Temprano de la Educación y Formación en

Castilla y León

María luz Martínez Seijo, Laura Rayón Rumayor y Juan Carlos Torrego Seijo

INE, Encuesta de Población Activa. Disponible en http://www.ine.es/jaxiBD/menu. do?divi=EPA\&his=1\&type $=\mathrm{db} \& \mathrm{~L}=0$ [consulta 2015, 27 de febrero]

Marina Torres, J.M., (2011). El abandono escolar, Revista de la Asociación de Inspectores de Educación de España, n 14, 5.

Mora Corral, A.J, (2010). Determinantes del abandono escolar en Cataluña: más allá del nivel socio-económico de las familias, Revista de Educación, número extraordinario, 171-190.

Portal de Educación, Educacyl. Becas, Ayudas y Subvenciones. Disponible en: http:// www.educa.jcyl.es/es/becasyayudas [consulta 2012, 13 de junio]

Poy Castro, R. (2010). Efectos del credencialismo y las expectativas sociales sobre el abandono escolar. Revista de Educación, número extraordinario, 147-169.

Presupuestos Generales de Castilla y León año 2009. (Tomo 11). Presupuestos Generales de la Comunidad de Castilla y León para el año 2012 (Tomo 11) Disponible en: http://www.jcyl.es/web/jcyl/Hacienda/es/Plantilla100/1138871919202/_/_[consulta 2012, 13 de junio]

Roca Cobo, E. (2010). El abandono temprano de la educación y la formación en España, Revista de Educación, número extraordinario, 31-62.

Serra Salamé, C. y Palaudárias Martí, J. (2010). Deficiencias en el seguimiento del abandono escolar y trayectorias de continuidad del alumnado de origen inmigrado, Revista de Educación, número extraordinario, 283-305

Vélaz de Medrano Ureta, C. y De Paz Higuera, A.B. (2010). Investigar sobre el deseo y la obligación de aprender en la sociedad del conocimiento. Revista de Educación, número extraordinario, 17-30.

VVAA (2006). Resumen ejecutivo sobre el estudio del abandono escolar prematuro, Revista de Educación, 341, 899-913. 